\title{
Solution Composition and Thermal Denaturation for the Production of Single- Stranded PCR Amplicons: Piperidine-Induced Destabilization of the DNA Duplex?
}

\author{
John B. Mangrum, Jason W. Flora, and David C. Muddiman ${ }^{+, \neq}$ \\ Department of Chemistry, Virginia Commonwealth University, Richmond, Virginia, USA
}

\begin{abstract}
Strategies to produce single-stranded PCR amplicons for detection by electrospray ionization Fourier transform ion cyclotron resonance mass spectrometry (ESI-FTICR-MS) were investigated using modified electrospray solutions and by thermally denaturing the duplex structures with a resistively heated electrospray ionization source. A synthetic 20-mer oligonucleotide annealed to its complementary strand was used as a model system for initial experiments. Electrospray solutions were altered by varying the relative proportion of aqueous phase in efforts to induce destabilization of the double helix. When the electrospray solution contains a $25 \%$ aqueous content, the 20 -mer oligonucleotide is detected in its double-stranded form. Increasing the proportion of aqueous phase in the electrospray solution to $60 \%$ destabilized the double helix, resulting in the detection of only single-stranded species. This strategy was extended to an 82-bp polymerase chain reaction (PCR) product derived from the human tyrosine hydroxylase gene (HUMTH01). In efforts to destabilize the 82-bp PCR product, electrospray solutions reaching $70 \%$ aqueous content were necessary to promote the detection of only single-stranded amplicons. Implementation of the resistively heated transfer line and an electrospray solution in which the oligonucleotide is on the threshold of duplex stability allowed for double-stranded and single-stranded species to be generated from the same ESI solutions at both ambient and elevated transfer line temperatures, respectively, without disruption of the electrospray process. The volatile base piperidine, present at $20 \mathrm{mM}$ concentrations in the electrospray solution, was found to play a critical role in the formation of single-stranded species at the higher aqueous percentages and a duplex destabilization mechanism has been proposed. (J Am Soc Mass Spectrom 2002, 13, 232-240) (c) 2002 American Society for Mass Spectrometry
\end{abstract}

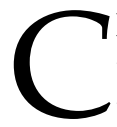
haracterization of DNA sequence variations in the post-genome era will require approaches that surpass existing methodologies in sensitivity, accuracy, and throughput. Recently, electrospray ionization [1] Fourier transform ion cyclotron resonance mass spectrometry (ESI-FTICR-MS) [2] has emerged as an ideal approach for genotyping length polymorphisms, namely, short tandem repeats (STRs) [3, 4]. STRs are the most valuable form of sequence variation for use as genetic markers because they are highly polymorphic $[5,6]$, occur approximately every 10-20 kb in the human genome [7-9], and are relatively easy to isolate using the polymerase chain reaction (PCR) $[7,8$, 10]. PCR amplicons generated from STR loci are typi-

Published online January 8, 2002

Address reprint requests to Dr. D. C. Muddiman, Department of Chemistry, Virginia Commonwealth University, 1001 West Main Street, P.O. Box 842006, Richmond, VA 23284, USA. E-mail: dcmuddim@vcu.edu

*Both researchers equally contriuted to these investigations. tAffiliate appointment in Biochemistry and Molecular Biophysics. $\ddagger$ Massey Cancer Center. cally observed as duplexes when using ESI-MS [3, 4, 11-23]. This soft ionization process enables retention of the Watson-Crick base pairing [24] of PCR amplicons.

ESI coupled with FTICR [25] has provided extraordinary accomplishments in the measurement of doublestranded PCR amplicons, with the largest detected being $500 \mathrm{bp}$ in length [17]. In addition, single acquisition mass spectra have been obtained from $5 \mathrm{nM}$ solutions of PCR amplicons [22]. The ultra-high mass accuracy of FTICR has been maximized with the use of a dual electrospray source [21, 26] allowing mass accuracy that routinely approaches the theoretical limit of $\pm 10 \mathrm{ppm}$ for large biomolecules $(>10 \mathrm{kDa})$ [27]. However, despite its powerful capabilities, the most challenging aspect of ESI-FTICR-MS is obtaining mass measurements with high enough mass accuracy to accurately genotype the length polymorphisms derived from complex STRs, which contain multiple repeat units, base insertions, and deletions [4, 28]. The difficulty of obtaining accurate mass measurements within duplex PCR amplicons arises from the fact that a base 
substitution, such as an $\mathrm{A} \rightarrow \mathrm{T}$ transversion, cannot be resolved by mass because of the $\mathrm{T} \rightarrow \mathrm{A}$ transversion in the complementary strand resulting in a mass difference of zero. Therefore, the production of singlestranded (ss) PCR amplicons is essential for the accurate characterization of base substitutions housed within STRs [3, 16].

Strategies for the routine production of singlestranded PCR products for mass spectrometry have included nozzle skimmer dissociation [29], biotinstreptavidin chemistry [30-36], and the DNA repair enzyme, lambda exonuclease $[3,16]$. It has been reported that the use of nozzle skimmer dissociation on duplex 16-mer oligonucleotides with a G-C content reaching $75 \%$ showed complete dissociation with no signs of covalent fragmentation [29]. Subsequently, in our laboratory, nozzle skimmer dissociation of a 20-mer with a G-C content of $70 \%$ promoted the formation of single-stranded amplicons; however, predominately duplex DNA remained in the spectrum and significant fragmentation was observed (data not shown). The production of single-stranded amplicons using biotinstreptavidin chemistry involves a biotinylated strand being bound to a magnetic bead and then denatured to remove the nonbiotinylated strand via strong base or thermal denaturation [30]. This method of producing single strands, although successful, has been shown to exhibit significant sample loss due to difficulties in removing the unbiotinylated strands [37]. Singlestranded PCR products have been successfully generated enzymatically in our lab via the use of lambda exonuclease which selectively digests the 5' phosphorylated strand of a duplex, leaving the complementary strand available for mass analysis [3, 16]. While this method has great applicability, it requires an additional enzymatic digestion step and incomplete phosphorylation of the primers renders the amplicon resistant to digestion by lambda exonuclease $[3,16]$. However, this method is advantageous because it allows researchers the ability to select which strand is generated following the digestion process as well as reduce the complexity of the mixture (e.g., multiplex PCR).

Efforts are being made to generate single-stranded amplicons on the fly prior to entering the mass spectrometer. Williams and co-workers have shown that thermal denaturation of oligonucleotides in the transfer line can be achieved via the use of a resistively heated coiled wire [38]. Generating single-stranded amplicons in the transfer line prior to the entrance into the mass spectrometer is advantageous because it eliminates the probability of sample contamination by reducing sample handling, as well as reducing the time frame for which samples can be prepared and analyzed. Our lab has recently developed a method using flow injection of PCR amplicons that will allow the analysis of $>2000$ genotypes per day $[3,23]$ greatly enhancing the genotyping capabilities of ESI-FTICR-MS.

Herein, we explore solution compositions and thermal denaturation during the ESI process in efforts to control the structural stability of DNA double helices. Experimental conditions have been developed which can promote either single-stranded or double-stranded moieties prior to entrance into the FTICR-MS.

\section{Experimental}

\section{Materials and Sample Preparation}

Complementary synthetic 20-mer oligonucleotides, 5'CAG CGT GCG CCA TCC TTC CC-3' and 5'-GGG AAG GAT GGC GCA CGC TG-3' were purchased from Midland Certified Reagent Company (Midland, TX). All oligonucleotides were desalted prior to ESI using the microdialysis apparatus previously described [18, 39] except with a molecular weight cut off (MWCO) of $18,000 \mathrm{Da}$ for the dialysis membrane. Concentrations for the stock solutions of oligonucleotides were quantified using UV-Vis spectrophotometry at $260 \mathrm{~nm}$ prior to dilution. The complementary 20-mers were annealed in a $1: 1$ molar ratio at $90^{\circ} \mathrm{C}$ for $2 \mathrm{~min}$ in their respective ESI solutions (vide infra) and slowly cooled to room temperature, with a final concentration of $4 \mu \mathrm{M}$ duplex. The 20-mer oligonucleotide duplex (average MW = $12,237.9 \mathrm{Da})$ has an estimated melting temperature $\left(T_{m}\right)$ of $71.6{ }^{\circ} \mathrm{C}$ which was calculated using eq 1 [40]:

$$
\begin{aligned}
T_{m}= & 22+1.46 I_{n} \text { where } I_{n}=2 \times(\# \text { GC base pairs }) \\
& + \text { (\# AT base pairs) }
\end{aligned}
$$

The $T_{m}$ is defined as the temperature at which one half of the duplex structure is in the denatured state. All other reagents were purchased from Sigma-Aldrich (St. Louis, MO); they were of the highest possible purity and used without purification.

\section{PCR Amplification}

The tetrameric repeating motif within HUMTH01 was amplified from K562 human genomic DNA (Promega, Madison, WI). This individual is homozygous for the 9.3 allele, thus yielding an 82 bp PCR amplicon with a theoretical average molecular weight of 50,535.47 Da for the double-stranded species. Each $50 \mu \mathrm{L}$ reaction consisted of $1 \times$ Pfu buffer, 1.5 units of Pfu (Promega, Madison, WI), $200 \mu \mathrm{M}$ of each dNTP (Promega), $0.5 \mu \mathrm{M}$ of each primer (Midland Certified Reagent Co., Midland, TX) and $100 \mathrm{ng}$ of template. Forward and reverse primer sequences along with themal cycler parameters are described in previously published work [3]. PCR amplicons were diluted with their respective electrospray solutions to give final concentrations of $2 \mu \mathrm{M}$.

\section{Mass Spectrometry}

ESI [1] offers a soft ionization method for transferring intact molecules from the solution phase to the gas phase without imparting fragmentation. Electrospray 


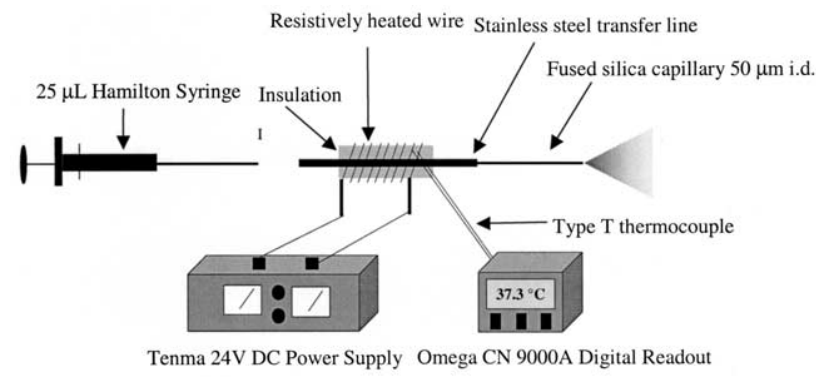

Figure 1. Schematic of ESI source with resistively heated transfer line.

ionization also has the ability to bestow multiple charge-states on large molecules resulting in $\mathrm{m} / \mathrm{z}$ ratios that fall within the $m / z$ range of most mass analyzers [1, 41]. The composition of ESI solutions can play an important role in charge-state distribution as well as ion intensities. ESI solutions will be delineated in the text as they were changed throughout the course of these experiments; however, imidazole was found to play an inconsequential role in duplex stability and therefore was not incorporated in later ESI solutions to simplify mechanistic arguments [42, 43]. All spectra were obtained using a modified IonSpec Corporation (Irvine, CA) FTICR mass spectrometer with a 4.7 tesla superconducting magnet (Cyromagnetics, Inc. Oak Ridge, $\mathrm{TN}$ ). The ESI source (Analytica of Branford, Inc, Branford, CT) was modified with a heated metal capillary [44], applying a constant temperature (allowing routine detection of noncovalent complexes without exception) for all experiments, and a micro-electrospray emitter. Emitter tips were hand pulled from a fused-silica capillary of $50 \mu \mathrm{m}$ to $10 \mu \mathrm{m}$ i.d. [45]. All samples were infused using a Harvard Syringe pump PHD 2000 (South Natick, MA) at an infusion rate of $3.3 \mathrm{~nL} / \mathrm{s}$.

All mass spectra were single acquisitions using $512 \mathrm{k}$ of data for 20-mer oligonucleotides and $32 \mathrm{k}$ of data for the 82-bp HUMTH01 PCR amplicon acquired at a 500 $\mathrm{kHz}$ ADC rate. Acquisitions involved two 1-s hexapole accumulations followed by gated trapping [46]. The unapodized data were zero filled two times and Fourier transformed prior to spectral analysis.

Figure 1 shows a schematic of the heated transfer line used for the thermal denaturation experiments. A DC voltage was applied using a Tenma $24 \mathrm{~V}$ DC power supply (Springboro, $\mathrm{OH}$ ) resistively heating the monochrome wire coiled around high temperature insulation. Real time temperature measurements were acquired using a type $\mathrm{T}$ thermocouple attached between the transfer line and the insulation. Temperatures were displayed on an Omega CN9000A readout (Stamford, CT).

\section{Results and Discussion}

Figure 2a shows an ESI-FTICR mass spectrum of the double-stranded 20 -mer $\left(T_{m}=71.6^{\circ} \mathrm{C}\right)$ using our traditional oligonucleotide ESI solution, $60 \%$ acetonitrile, $20 \%$ isopropyl alcohol, $20 \%$ aqueous phase with final

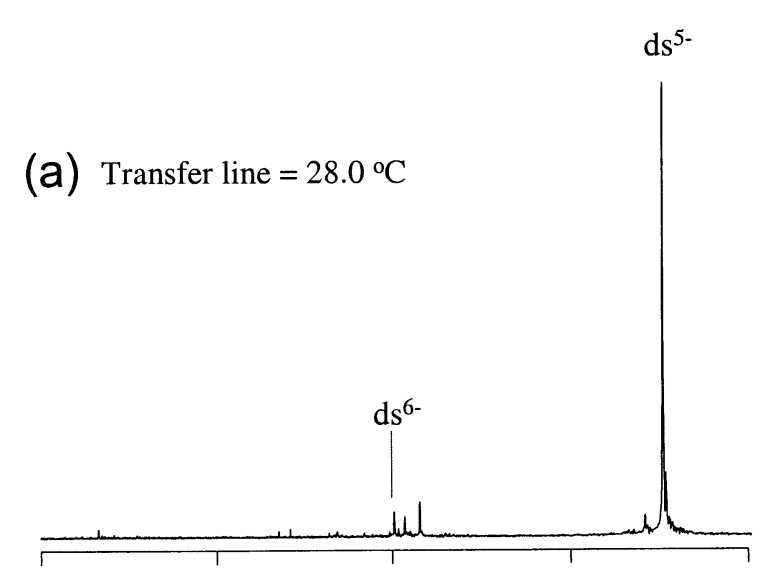

(b) Transfer line $=88.4^{\circ} \mathrm{C}$

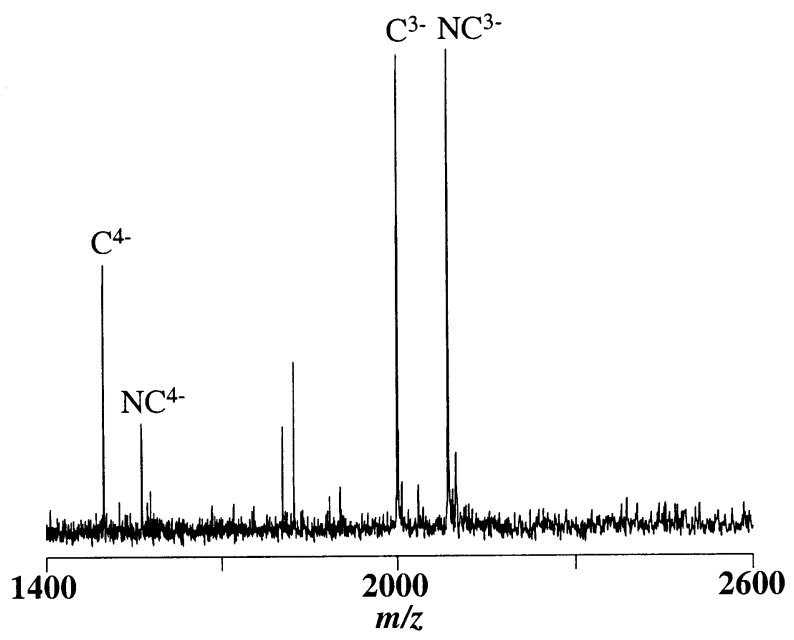

Figure 2. ESI-FTICR mass spectra of duplex 20-mer oligonucleotide using an ESI solution consisting of $60 \%$ acetonitrile, $20 \%$ isopropyl alcohol, $2 \mathrm{mM}$ ammonium acetate with final concentration of $20 \mathrm{mM}$ piperidine and imidazole. (a) Mass spectrum of the 20-mer duplex observed at ambient temperature $\left(28^{\circ} \mathrm{C}\right)$. (b) Mass Spectrum of the 20-mer duplex, illustrating that ssDNA is observed when temperature of the heated metal transfer line $\left(88.4^{\circ} \mathrm{C}\right)$ is raised significantly above its $T_{m}$. All charge-states, coding (C) and noncoding (NC) strands are labeled as well as double-stranded DNA (ds).

concentrations of $2 \mathrm{mM}$ ammonium acetate and $20 \mathrm{mM}$ piperidine and imidazole [3, 4, 16-23]. Figure $2 \mathrm{~b}$ shows the result of using the resistively heated transfer line providing complete thermal denaturation of the duplex DNA (20-mer) as it was introduced into the orifice of the mass spectrometer. However, the elevated temperatures required to destabilize the hydrogen bonding between the nucleobases of the 20-mer resulted in a highly unstable electrospray, causing a significant reduction of absolute ion intensity and lack of reproducibility.

In efforts to circumvent the instability and low signal-to-noise ratio during the thermal denaturation, the aqueous character of the electrospray solutions was 


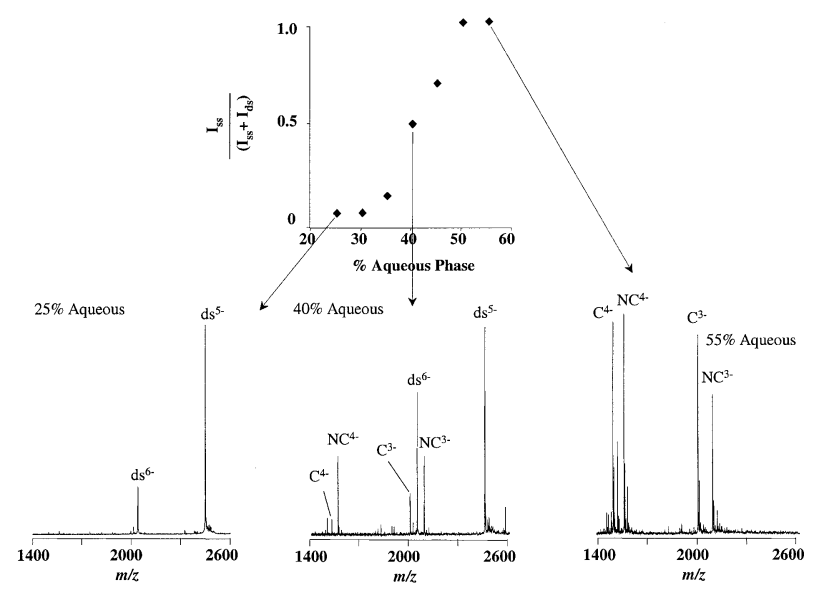

Figure 3. Plot of relative ion intensity of single-stranded 20-mer versus percent aqueous content. Representative spectra are shown using ESI solution with 25,40 , and $55 \%$ aqueous content. $\left(\mathrm{I}_{\mathrm{ss}}=\right.$ intensity of single-stranded species), $\left(\mathrm{I}_{\mathrm{ds}}=\right.$ intensity of doublestranded species).

increased. However, we observed that as the aqueous content of the ESI solutions was increased to higher proportions, the dsDNA became denatured and singlestranded DNA (ssDNA) was detected. Figure 3 shows ESI-FTICR mass spectra of the 20-mer DNA duplex at ambient temperature with various aqueous proportions including a plot of relative ion intensity of the singlestranded oligonucleotides (intensity of single stranded species, $I_{s s}$ divided by total intensity, $I_{s s}+I_{d s}$ ) versus the percent aqueous content. ESI solutions consisting of a $25 \%$ aqueous content resulted in retention of the duplex structure. The 20-mer oligonucleotide in an electrospray solution comprised of a $40 \%$ aqueous phase exists in both duplex and single-stranded forms. As the aqueous phase was increased to $50-60 \%$, the 20-mer DNA duplex became destabilized and only single-stranded oligonucleotides are observed.

The signal-to-noise ratio of the single-stranded mass spectral data presented in Figures $2 b$ and 3 appears to have been compromised by the higher aqueous content. This is attributed to the following: (1) The total ion intensity is distributed among four species $\left(\mathrm{C}^{4-}, \mathrm{NC}^{4-}\right.$, $\mathrm{C}^{3-}$, and $\mathrm{NC}^{3-}$ ) at high aqueous content (55\%) rather than two species $\left(\mathrm{ds}^{6-}\right.$ and $\mathrm{ds}^{5-}$ ) at low aqueous content $(25 \%)$; (2) the surface tension is higher when one increases the aqueous content of the solution; however, we kept the heated metal capillary temperature constant which likely resulted in lower ion production; and (3) the FTICR signal scales linear with charge; thus, the lower charged (i.e., single-stranded species) induce less image current than the duplex species. The average charge-state for the duplex species also increases with increasing aqueous content (Figure 3). This is attributed to: (1) The higher aqueous content droplets will accommodate a higher charge density, therefore imparting more charge onto the oligonucleotide structure; and (2) the apparent $\mathrm{pH}$ of the solution increases slightly with increasing aqueous content. An increase in
$\mathrm{pH}$ decreases the relative proportion of protonated piperidine which is known to reduce charge-states when in its protonated form [43].

The single-stranded products detected in Figure 3 show no evidence of fragmentation and represent individual intact strands. This is consistent with the work done by Gabelica et al. in which they used a higher aqueous solution for the nozzle skimmer dissociation of 16-mer duplexes [29]; compared to the data presented in Figure 3, their duplexes were destabilized to a greater extent because of the higher aqueous content allowing for the conversion to the single-stranded state using collisional heating in the nozzle-skimmer region. Our previous work with nozzle skimmer dissociation (vide supra) utilized a solution composition that was conducive to duplex stability (i.e., $20 \%$ aqueous content; c.f.g. Figures $2 b$ and 3 ) and therefore resulted in higher amounts of covalent fragmentation.

Based on the initial findings with the 20-mer model system, an 82-bp PCR amplicon was electrosprayed using ESI solutions that were comprised of 50, 60, and $70 \%$ aqueous phase. Higher aqueous contents were chosen based on the number of base pairs and estimated $T_{m}$ for the PCR amplicon for a specific ionic strength. The theoretical $T_{m}$ for the amplicon was generated using eq 2 [40]:

$$
\begin{aligned}
T_{m}= & 81.5+\left[16.6\left(\log \left[X^{+}\right]\right)\right]+[0.41(\% \mathrm{GC})] \\
& -\left[\frac{500}{\# \text { of base pairs }}\right]
\end{aligned}
$$

Eq 2 is only valid for monovalent cation concentrations, represented by $\left[\mathrm{X}^{+}\right]$, in the range of $0.01-0.4 \mathrm{M}$ and the DNA duplex of interest must contain a GC percentage between $30-75 \%$ [40]. The sodium ion concentration was assumed to be zero since all samples were thoroughly dialyzed to remove the adduction of salt (i.e., the sodium ion concentration would be a minor contributor to the overall ionic strength of the solution). However, the ESI solutions also contain ammonium ions and protonated piperidine (vide infra). The $T_{m}$ was calculated using protonated piperidine concentrations as determined by $\alpha$-values for a given $\mathrm{pH}$ (vide infra). Figure $4 \mathrm{a}$ shows an ESI-FTICR mass spectrum of a stable DNA duplex obtained from a $50 \%$ aqueous ESI solution $\left(\mathrm{pH}=11.1 ; T_{m}=58.5^{\circ} \mathrm{C}\right)$. Figure $4 \mathrm{~b}$ shows a mass spectrum of the 82-bp PCR amplicon obtained from an ESI solution containing $60 \%$ aqueous phase, resulting in only a marginal amount of single-stranded species $\left(\mathrm{pH}=11.2 ; T_{m}=57.6{ }^{\circ} \mathrm{C}\right)$. Figure $4 \mathrm{c}$ shows a mass spectrum of a completely denatured $82-b p$ PCR product obtained from an ESI solution with $70 \%$ aqueous phase $\left(\mathrm{pH}=11.3 ; T_{m}=56.9^{\circ} \mathrm{C}\right)$. Thus, as we increased the aqueous character of the ESI solution, the $\mathrm{pH}$ also increased which decreased the amount of protonated piperidine and therefore decreased the ionic strength of the solution. However, over this narrow 


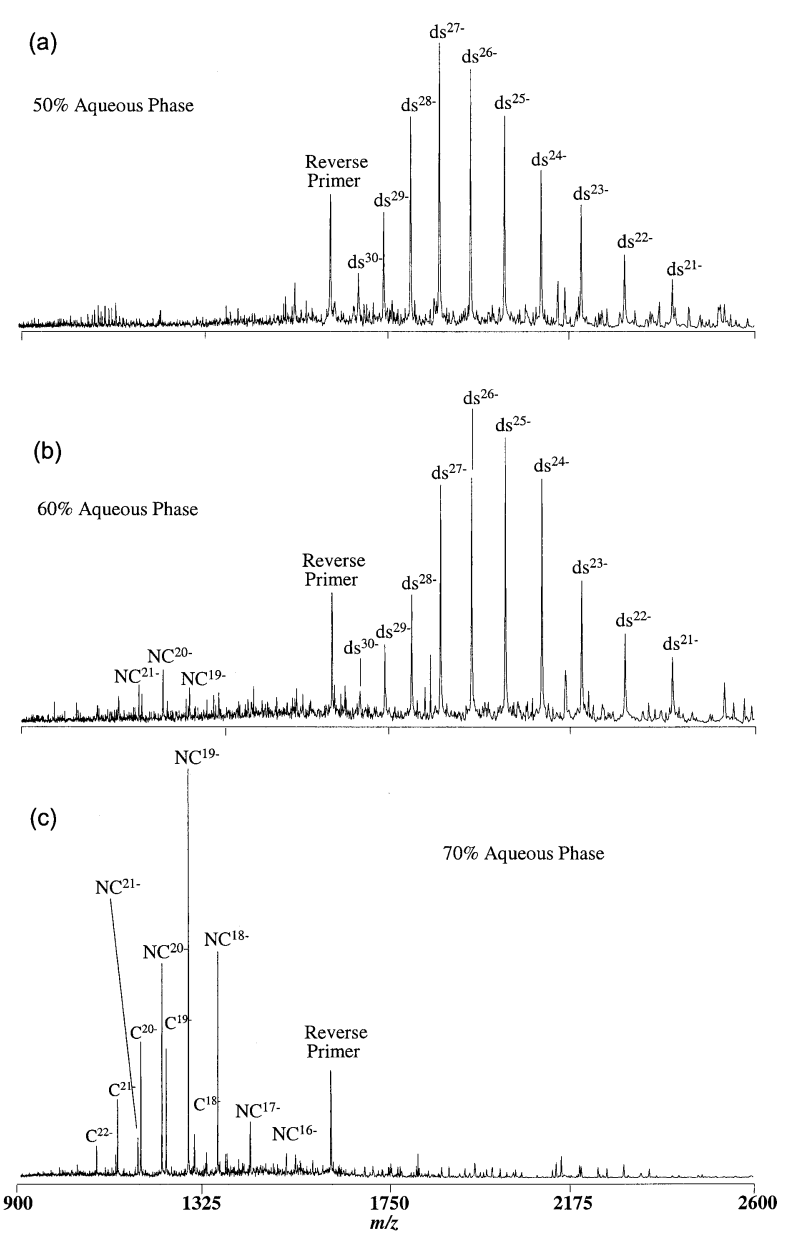

Figure 4. ESI-FTICR mass spectra of an 82-bp PCR amplicon at ambient temperature using ESI solutions of varying aqueous content. Solutions consisted of $20 \mathrm{mM}$ piperidine, $20 \mathrm{mM}$ imidazole, and $2 \mathrm{mM}$ ammonium acetate with (a) $50 \%$ aqueous ( $\mathrm{pH}=$ $11.1),($ b) $60 \%$ aqueous $(\mathrm{pH}=11.2)$, and (c) $70 \%$ aqueous $(\mathrm{pH}=$ 11.3).

range of ionic strengths, the melting temperature only changed by $1.6^{\circ} \mathrm{C}$ suggesting that additional factors play a role in the DNA duplex stability such as the degree of hydration and the hydrogen bonding capability of neutral piperidine.

In efforts to thermally control the state of the species (ssDNA or dsDNA) observed in the mass spectrum, resistive heating of the transfer line using the $60 \%$ aqueous ESI solution $\left(\mathrm{pH}=11.2 ; T_{m}=57.6^{\circ} \mathrm{C}\right)$ was chosen since it appears to be on the threshold of the 82-bp duplex stability (see Figure $4 \mathrm{~b}$ ). Figure 5shows the resulting mass spectra of the 82-bp PCR product in an ESI solution of $60 \%$ aqueous content while increasing the solution temperature via the resistively heated transfer line. This method allows one to control the state of the amplicon during the same ESI experiment. Figure 5 also shows a plot of the relative ion intensity of the single stranded state versus the temperature of the heated transfer line. At a transfer line temperature of $28^{\circ} \mathrm{C}$, the amplicon that is detected is primarily dsDNA. As the transfer line temperature is raised to

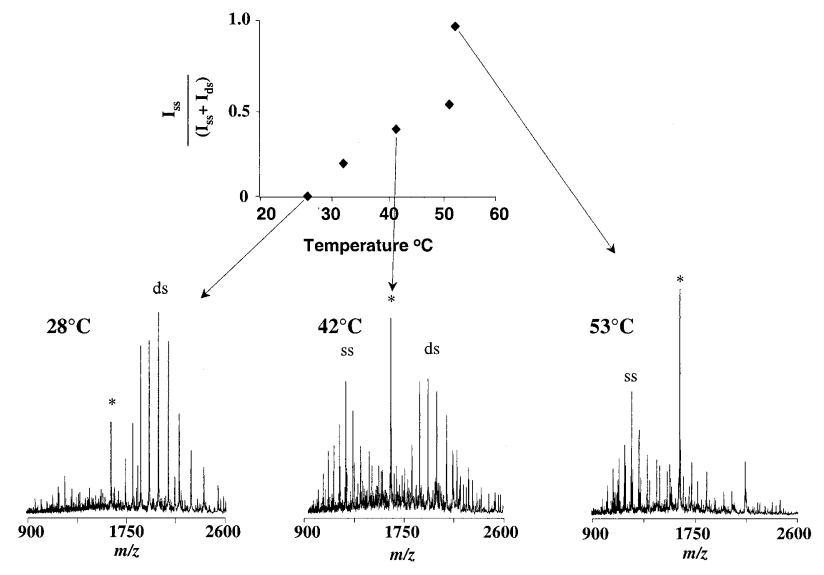

Figure 5. Plot of relative ion intensity of an 82-bp PCR product versus temperature of resistively heated transfer line. ESI-FTICR mass spectra obtained from a $60 \%$ aqueous ESI solution illustrating the production of single-stranded species with variable transfer line temperatures. ${ }^{*}=$ reverse primer $)$.

$42{ }^{\circ} \mathrm{C}$, the PCR amplicon is observed in both the doublestranded and single-stranded states. Increasing the temperature of the transfer line to $53^{\circ} \mathrm{C}$ resulted in the production of single-stranded moieties with only minimal amounts of duplex remaining. The PCR amplicon destabilized at a temperature well below the estimated $T_{m}\left(57.6{ }^{\circ} \mathrm{C}\right)$, which is consistent with our observation that with a higher aqueous phase in the electrospray solution, the $T_{m}$ decreases (see Figures 3 and 4 ).

It was observed that with the increase in aqueous content of the ESI solutions, the apparent $\mathrm{pH}$ of each solution also increased. This indicated that the $\mathrm{pH}$ of the electrospray solution might play a crucial role in DNA duplex stability. Piperidine, a strong base $(\mathrm{pKa}=$ 11.1), raises the $\mathrm{pH}$ of the solutions as well as reduces the alkali metal adduction [43]. Based on an aqueous phase $\alpha$-plot for piperidine, at $\mathrm{pH}$ values below its $\mathrm{pKa}$ (11.1), the protonated form of piperidine is predominant in the ESI solution. At $\mathrm{pH}$ values above the $\mathrm{pKa}$, piperidine resides primarily in its neutral state. In accord with the $\alpha$-plot, $\alpha_{0}=0.61$ for the protonated form of piperidine and $\alpha_{1}=0.39$ for neutral piperidine, for the $20 \%$ aqueous ESI solution (apparent $\mathrm{pH}=10.9$ ). The corresponding $\alpha$ values for the 55\% aqueous ESI solution (apparent $\mathrm{pH}=11.3$ ) are $\alpha_{0}=0.43$ and $\alpha_{1}=$ 0.57 . For the 20-mer oligonucleotide, these two aqueous percentages and their corresponding $\alpha$ values illustrate that the percentage of protonated and neutral piperidine in the solution seemed to play a decisive role as to whether the 20-mer resides in the double-stranded or single-stranded form.

Figure 6 shows the ESI-FTICR mass spectra of a 20-mer electrosprayed from various ESI solutions, none of which contained imidazole. Figure $6 a$ shows an ESI-FTICR mass spectrum of the 20-mer electrosprayed from a $20 \%$ aqueous solution in which the doublestranded form is observed as expected for this $\mathrm{pH}$. Figure $6 \mathrm{~b}$ shows a mass spectrum where the ESI solu- 
(a)

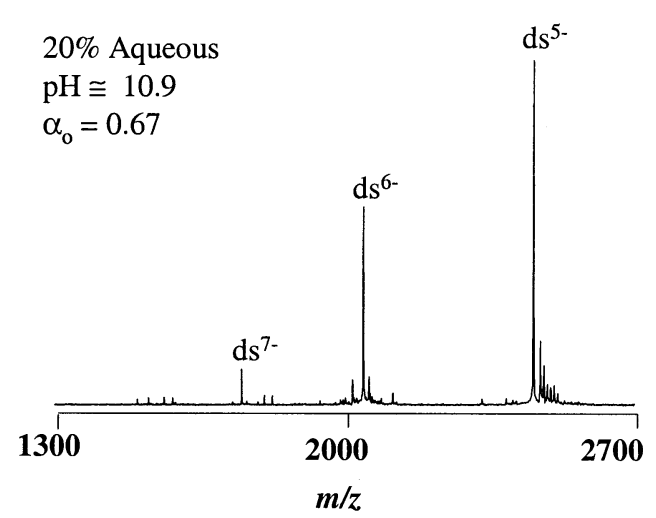

(b)
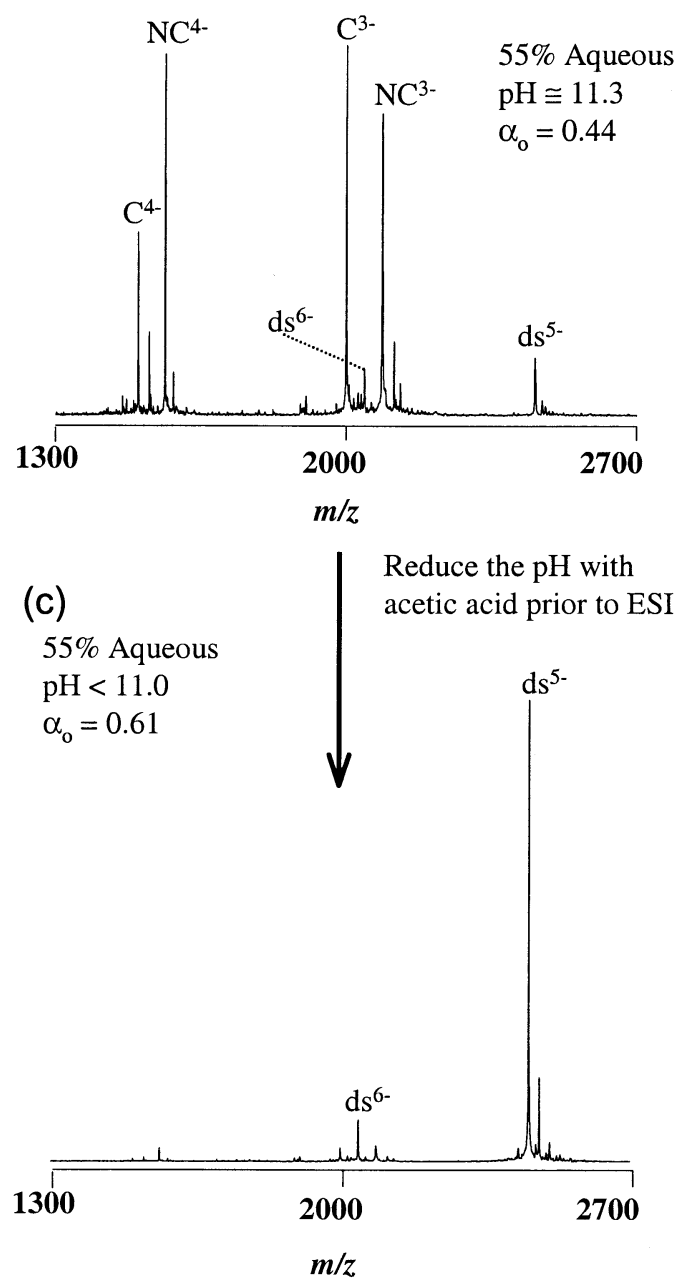

Figure 6. ESI-FTICR mass spectra of the 20-mer oligonucleotide duplex at ambient temperature showing that the stability of the DNA duplex is affected by fraction of protonated piperidine in the ESI solution. (a) With a $20 \%$ aqueous solution, apparent $\mathrm{pH}$ of 10.9, the duplex structure is retained. (b) At $55 \%$ aqueous content, apparent $\mathrm{pH}$ of 11.3 , destabilization of the helical conformation is promoted. (c) In the same $55 \%$ aqueous solution with the apparent $\mathrm{pH}$ adjusted to slightly less than 11.0 by the addition of acetic acid, the double-stranded conformation is retained (the $\mathrm{pH}$ is below the pKa of piperidine).

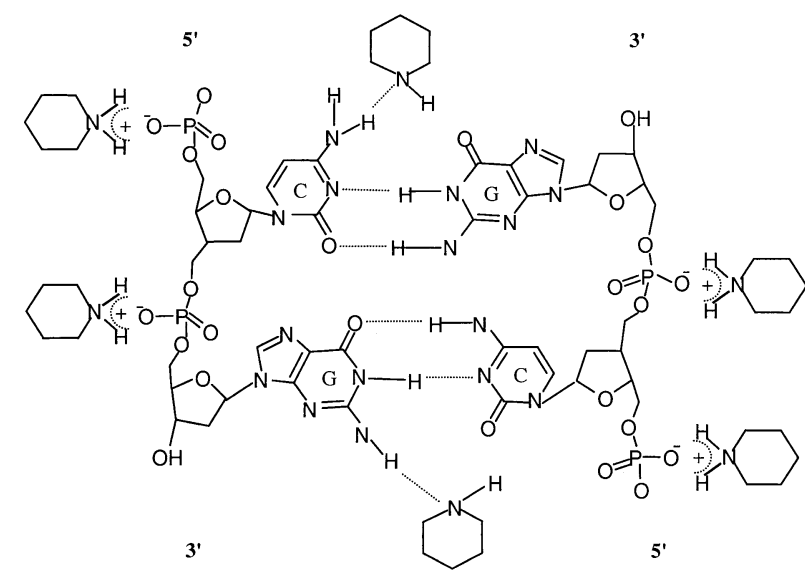

Scheme 1

tion is $55 \%$ aqueous inducing the production of singlestranded moieties. Figure $6 \mathrm{c}$ shows the mass spectrum using a $55 \%$ aqueous ESI solution where apparent $\mathrm{pH}$ has been slightly lowered below 11.0 with the addition of a small amount of acetic acid. While all singlestranded species are present in the ESI solution at a $\mathrm{pH}$ greater than the $\mathrm{pKa}$ of piperidine, the duplex state of the 20-mer is retained when the apparent $\mathrm{pH}$ of the same ESI solution is reduced below the pKa of piperidine. The average charge-state for the duplexes shown in Figure $6 a$ with $6 c$ are 5.6 and 5.1, respectively. This decrease in the average charge-state is due to the approximately $6 \%$ increase in protonated piperidine which reduces charge-states [43].

Scheme 1 illustrates the proposed mechanism for piperidine interaction based upon the experimental data and the $\alpha$-plot for piperidine. At $\mathrm{pH}$ values below the $\mathrm{pKa}$ of piperidine, the excess of protonated piperidine acts as a cation and electrostatically binds to the phosphate backbone neutralizing the repulsive negative charges and thus creating an effect similar to peptide nucleic acid (PNA) duplex stability where Coulombic repulsions have been eliminated [47]. This effect is analogous to the $\mathrm{Na}^{+}$cation binding to the negatively charged phosphate backbone stabilizing the duplex by reducing the electrostatic repulsion $[48,49]$. At $\mathrm{pH}$ values above the suggested pKa of piperidine, the excess of neutral piperidine can hydrogen bond with the nucleobases destabilizing the double helix analogous to the denaturant formamide [50].

Further evidence in support of this mechanism was accomplished by studying the melting temperatures of the 20-mer duplex $\left(T_{m}=71.6^{\circ} \mathrm{C}\right)$ in two ESI solutions with slightly different apparent $\mathrm{pH}$ values. Figure 7 is a plot of relative ion intensity of the single-stranded 20-mer versus the temperature of the resistively heated transfer line for two $30 \%$ aqueous ESI solutions at $\mathrm{pH}$ 10.9 and 10.7; the latter $\mathrm{pH}$ was decreased by the addition of acetic acid. When electrosprayed from an ESI solution of $\mathrm{pH} 10.9$ at room temperature $\left(28^{\circ} \mathrm{C}\right)$, the mass spectrum shows duplex DNA. Heating the trans- 


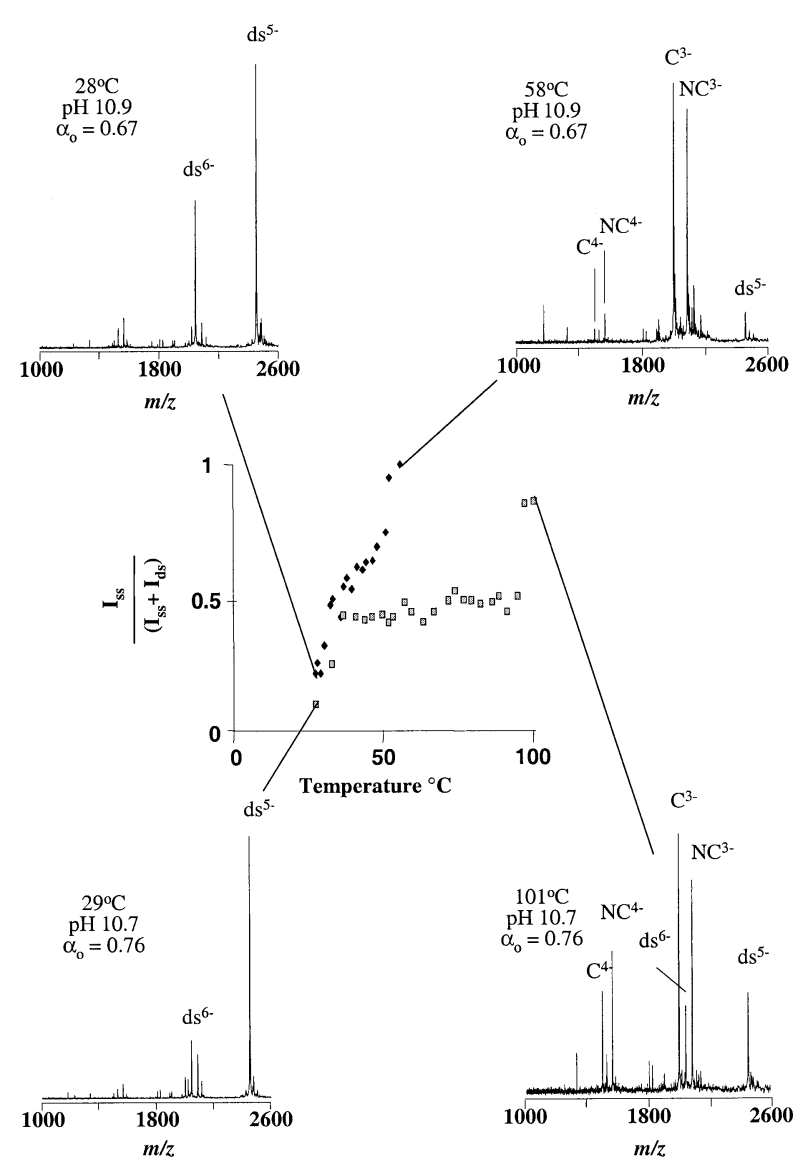

Figure 7. ESI-FTICR mass spectra of the 20-mer oligonucleotide duplex in 30\% aqueous ESI solution at $\mathrm{pH} 10.9$ (filled diamond) and $\mathrm{pH} 10.7$ (filled square). A plot of relative ion intensity of the single-stranded species versus temperature of the transfer line is shown.

fer line to $58{ }^{\circ} \mathrm{C}$ results in almost complete denaturation of the 20-mer, as shown in the mass spectrum, with only minimal duplex structure remaining. However, the effect of lowering the $\mathrm{pH}$ with acetic acid (i.e., producing more protonated piperidine, $\mathrm{pH}=10.7)$, clearly increases the stability of the duplex structure as well as increasing the $T_{m}$ of the 20-mer oligonucleotide. Temperatures reaching $100{ }^{\circ} \mathrm{C}$ were necessary to achieve dissociation of the duplex structure. Analogous to PNA-PNA duplex stability [47], the oligonucleotide electrosprayed from a solution of lower $\mathrm{pH}$ (i.e., more protonated piperidine) stabilizes the anionic phosphate backbone of the double helix and melts at a temperature that is roughly twice that of the duplex electrosprayed from a higher $\mathrm{pH}$ solution. The decrease in the $\mathrm{pH}$ from 10.9 to 10.7 is also reflected in the charge-states of the duplex structures at ambient temperature; when the concentration of protonated piperidine increases by $9 \%$, the average charge-state decreases.

In addition to the role piperidine plays on duplex stability, the degree of duplex hydration also contributes to the destabilization effects on the duplex structure. Swelling of the DNA duplex occurs as the double helix becomes completely hydrated with approximately 15-20 water molecules per nucleobase [51]. This swelling would allow the neutral piperidine easier accessibility to the nucleobases and promote the disruption of the hydrogen bonding as described by the proposed mechanism.

Due to the relatively complex ESI solution compositions and the numerous factors that play a role in DNA duplex stability (e.g., ionic strength which is $\mathrm{pH}$ dependent, aqueous content, temperature), more investigations are warranted to fully test the proposed mechanism. Currently, we are carrying out a titration experiment of piperidine concentration relative to aqueous content and accurately determining ionic strength (using doubly-dialyzed solutions to reduce alkali metal concentrations to a level in which they will no longer play a role) by carefully measuring the conductivity of each ESI solution. These experiments should enable us to develop stronger mechanistic arguments in the near future.

\section{Conclusions}

We have demonstrated the ability to routinely generate single-stranded amplicons prior to entrance into the mass spectrometer. Stability of the DNA duplex has been shown in this research to be affected by many parameters including temperature, ionic strength, and aqueous content of the ESI solution, all of which play a combined role in stabilizing or destabilizing the helical structure of DNA. Single-stranded moieties can be routinely generated from a double-stranded PCR product. The resistively heated metal transfer line, used to thermally dissociate the duplex structures, allowed control of which physical state of the DNA was observed. The state of piperidine as controlled by $\mathrm{pH}$ in the aforementioned ESI solution was found to affect duplex stability by disrupting the hydrogen bonds between the nucleobases or by stabilizing the phosphate backbone as described by the proposed mechanism. Higher aqueous contents, resulting in DNA swelling, of the ESI solutions enable neutral piperidine to interact and disrupt the hydrogen bonds found within the groove of the helical DNA structure. With the ability to continually and rapidly generate single-stranded amplicons, the potential role of automated high throughput genotying of complex STRs by ESIFTICR-MS becomes feasible.

\section{Acknowledgments}

The authors would like to thank reviewers of this manuscript for their insightful comments. The authors would also like to thank Ms. Allison P. Null for her help in manuscript preparation and Dr. Sarah Rutan for helpful discussions. We also gratefully acknowledge the National Institutes of Health (R01HG02159) and the Department of Chemistry (Mary E. Kapp Foundation), Virginia Commonwealth University, for their generous financial support. 


\section{References}

1. Fenn, J. B.; Mann, M.; Meng, C. K.; Wong, S. F.; Whitehouse, C. M. Electrospray Ionization for Mass Spectrometry of Large Biomolecules. Science 1989, 246, 64-71.

2. Comisarow, M. B.; Marshall, A. G. Fourier Transform Ion Cyclotron Resonance Mass Spectrometry. Chem. Phys. Lett. 1974, 25, 282-283.

3. Null, A. P.; Hannis, J. C.; Muddiman, D. C. Genotyping of Simple and Compound Short Tandem Repeat Loci Using Electrospray Ionization Fourier Transform Ion Cyclotron Resonance Mass Spectrometry. Anal. Chem. 2001, 73, 4514-4521.

4. Null, A. P.; Muddiman, D. C. Perspectives on the Use of Electrospray Ionization Fourier Transform Ion Cyclotron Resonance Mass Spectrometry for Short Tandem Repeat Genotyping in the Post-Genome Era. J. Mass Spectrom. 2001, 36, 589-606.

5. Weber, J. L. Human DNA Polymorphisms and Methods of Analysis. Curr. Opin. Biotechnol. 1990, 1, 166-71.

6. Weber, J. L.; Wong, C. Mutation of Human Short Tandem Repeats. Hum. Mol. Gen. 1993, 2, 1123-1128.

7. Weber, J. L.; May, P. E. Abundant Class of Human DNA Polymorphisms Which Can be Typed Using the Polymerase Chain Reaction. Am. J. Hum. Genet. 1989, 44, 388-396.

8. Litt, M.; Luty, J. A. A Hypervariable Microsatellite Revealed by in vitro Amplification of a Dinucleotide Repeat Within the Cardiac Muscle Actin Gene. Am. J. Hum. Genet. 1989, 44, 397-401.

9. Edwards, A.; Civitello, A.; Hammond, H. A.; Caskey, T. C. DNA Typing and Genetic Mapping with Trimeric and Tetrameric Tandem Repeats. Am. J. Hum. Genet. 1991, 49, 746-756.

10. Mullis, K.; Faloona, F. Specific Synthesis of DNA in vitro via Polymerase Catalyzed Chain Reaction. Methods Enzymol. 1987, 155, 335.

11. Bayer, E.; Bauer, T.; Schmeer, K.; Bleicher, K.; Maler, M.; Gaus, H. J. Analysis of Double-Stranded Oligonucleotides by Electrospray Mass Spectrometry. Anal. Chem. 1994, 66, 3858-3863.

12. Doktycz, M. J.; Habibigoudarzi, S.; Mcluckey, S. A. Accumulation and Storage of Ionized Duplex DNA Molecules in a Quadrupole Ion Trap. Anal. Chem. 1994, 66, 3416-3422.

13. Aaserud, D. J.; Kelleher, N. L.; Little, D. P.; McLafferty, F. W. Accurate Base Composition of Double-Strand DNA by Mass Spectrometry. J. Am. Soc. Mass Spectrom. 1996, 7, 1266-1269.

14. Greig, M. J.; Gaus, H. J.; Griffey, R. H. Negative Ionization Micro Electrospray Mass Spectrometry of Oligonucleotides and Their Complexes. Rapid Commun. Mass Spectrom. 1996, 10, 47-50.

15. Ganem, B.; Li, Y. T.; Henion, J. D. Detection of Oligonucleotide Duplex Forms by Ion-Spray Mass-Spectrometry. Tetrahedron Lett 1993, 34, 1445-1448.

16. Null, A. P.; Hannis, J. C.; Muddiman, D. C. Preparation of Single-Stranded PCR Products for Electrospray Ionization Mass Spectrometry Using the DNA Repair Enzyme Lambda Exonuclease. The Analyst (Special Issue on Biological Mass Spectrometry) 2000, 125, 619-625.

17. Muddiman, D. C.; Null, A. P.; Hannis, J. C. Precise Mass Measurement of a Double-Stranded 500 Base-Pair (309 kDa) Polymerase Chain Reaction Product by Negative Ion Electrospray Ionization Fourier Transform Ion Cyclotron Resonance Mass Spectrometry. Rapid Commun. Mass Spectrom. 1999, 13, 1201-1204.

18. Hannis, J. C.; Muddiman, D. C. Characterization of a Microdialysis Approach for the Preparation of PCR Products for ESI-MS Using ICP-AES and On-Line UV-Vis Detection. Rapid Commun. Mass Spectrom. 1999, 13, 323-330.

19. Hannis, J. C.; Muddiman, D. C. Accurate Characterization of the Tyrosine Hydroxylase Forensic Allele 9.3 Through Development of Electrospray Ionization Fourier Transform Ion Cyclotron Resonance Mass Spectrometry. Rapid Commun. Mass Spectrom. 1999, 13, 954.
20. Hannis, J. C.; Muddiman, D. C.; Null, A. P. Advances in Nucleic Acid and Protein Analyses, Manipulation, and Sequencing. SPIE-Bios Meeting: San Jose, CA, 2000; pp 36-47.

21. Hannis, J. C.; Muddiman, D. C. A Dual Electrospray Ionization Source Combined with Hexapole Accumulation to Achieve High Mass Accuracy of Bioploymers in FTICR Mass Spectrometry. J. Am. Soc. Mass Spectrom. 2000, 11, 876-883.

22. Hannis, J. C.; Muddiman, D. C. Detection of Double-Stranded PCR Amplicons at the Attomole Level Electrosprayed from Low Nanomolar Solution Using FT-ICR Mass Spectrometry. Fresenius J. Anal. Chem. 2001, 369, 246-251.

23. Hannis, J. C.; Muddiman, D. C. Genotyping Short Tandem Repeats Using Flow Injection and ESI FT-ICR-MS. Rapid Commun. Mass Spectrom. 2001, 15, 348-350.

24. Watson, J. D.; Crick, F. H. C. Molecular Structure of Nucleic Acids. Nature 1953, 171, 737-738.

25. Henry, K. D.; Williams, E. R.; Wang, B. H.; McLafferty, F. W.; Shabanowitz, J.; Hunt, D. F. Fourier-Transform Mass Spectrometry of Large Molecules by Electrospray Ionization. Proc. Natl. Acad. Sci. U.S.A. 1989, 86, 9075-9078.

26. Flora, J. W.; Hannis, J. C.; Muddiman, D. C. High-Mass Accuracy of Product Ions Produced by SORI-CID Using a Dual Electrospray Ionization Source Coupled with FTICR Mass Spectrometry. Anal. Chem. 2001, 73, 1247-1251.

27. Beavis, R. C. Chemical Mass of Carbon in Proteins. Anal. Chem. 1993, 65, 496-497.

28. Urquhart, A.; Kimpton, C. P.; Downes, T. J.; Gill, P. Variation in Short Tandem Repeat Sequences-A Survey of Twelve Microsatellite Loci for Use as Forensic Identification Markers. Int. J. Leg. Med. 1994, 107, 13-20.

29. Gabelica, V.; DePauw, E. Comparison Between Solution-Phase Stability and Gas-Phase Kinetic Stability of Oligodeoxynucleotide Duplexes. J. Mass Spectrom. 2001, 36, 397-402.

30. Ross, P. L.; Lee, K.; Belgrader, P. Discrimination of SingleNucleotide Polymorphisms in Human DNA Using Peptide Nucleic Acids Probes Detected by MALDI-TOF Mass Spectrometry. Anal. Chem. 1997, 69, 4197-4202.

31. Uhlen, M. Magnetic Separation of DNA. Nature 1989, 340, 733-744.

32. Tang, K.; Fu, D.; Kotter, S.; Cotter, R. J.; Cantor, C. R.; Koster, H. Matrix-Assisted Laser Desorption/Ionization Mass Spectrometry of Immobilized Duplex DNA Probes. Nucleic Acids Res. 1995, 23, 3126-3131.

33. Chou, C. W. Bingham, S.; Williams, P. Affinity Methods for Purification of DNA Sequence Reactions Products for Mass Spectrometric Analysis. Rapid Commun. Mass Spectrom. 1996, 10, 1410-1414.

34. Koster, H.; Tang, K.; Fu, D. J.; Braun, A.; Boom, D. V. D.; Smith, C. L.; Cotter, R. J.; Cantor, C. R. A Strategy for Rapid and Efficient DNA Sequencing by Mass Spectrometry. Nat. Biotechnol. 1996, 14, 1123-1586.

35. Jurinke, C.; van der Boom, D.; Jacob, A.; Tang, K.; Worl, R.; Koster, H. Analysis of Ligase Chain Reaction Products via Matrix Assisted Laser Desorption Ionization Time-of-Flight Mass Spectrometry. Anal. Biochem. 1996, 237, 174-181.

36. Jurinke, C. van den; Boom, D.; Collazo, V.; Luchow, A.; Jacob, A.; Koster, H. Recovery of Nucleic Acids form Immobilized Biotin-Streptavidin Complexes Using Ammonium Hydroxide and Applications in MALDI-TOF Mass Spectrometry. Anal. Chem. 1997, 69, 904-910.

37. Krahmer, M. T.; Johnson, Y. A.; Walters, J. J.; Fox, K. F.; Fox, A. F.; Nagpal, M. Electrospray Quadrupole Mass Spectrometry Analysis of Model Oligonucleotides and Polymerase Chain Reaction Products: Determination of Base Substitutions, Nucleotide Additions/Deletions, and Chemical Modifications. Anal. Chem. 1999, 71, 2893-2900. 
38. Jurchen, J. C.; Rodriquez-Cruz, S. E.; Williams, E. R. A Comparison of the Thermal Stability of High Order DNA Structures in Solution and in the Gas-Phase. Proceedings of the 47th Annual ASMS Conference on Mass Spectrometry and Allied Topics; Dallas, TX, 1999; pp 2599-2600.

39. Liu, C. L.; Wu, Q. Y.; Harms, A. C.; Smith, R. D. On-Line Microdialysis Sample Cleanup for Electrospray Ionization Mass-Spectrometry of Nucleic Acid Samples. Anal. Chem. 1996, 68, 3295-3299.

40. Strachan, T.; Read, A. P. Human Molecular Genetics 2. John Wiley \& Sons, Inc: New York, 1999.

41. Kebarle, P.; Tang, L. From Ions in Solution to Ions in the Gas Phase-The Mechanism of Electrospray Mass Spectrometry. Anal. Chem. 1993, 65, A972-A986.

42. Greig, M.; Griffey, R. H. Utility of Organic Bases for Improved Electrospray Mass Spectrometry of Oligonucleotides. Rapid Commun. Mass Spectrom. 1995, 9, 97-102.

43. Muddiman, D. C.; Cheng, X. H.; Udseth, H. R.; Smith, R. D. Charge-State Reduction with Improved Signal Intensity of Oligonucleotides in Electrospray Ionization Mass Spectrometry. J. Am. Soc. Mass Spectrom. 1996, 7, 697-706.

44. Chowdhury, S. K.; Katta, V.; Chait, B. T. An ElectrosprayIonization Mass Spectrometer with New Features. Rapid. Commun. Mass Spectrom. 1990, 4, 81-87.
45. Hannis, J. C.; Muddiman, D. C. Nanoelectrospray Mass Spectrometry Using Non-Metalized, Tapered (50-10 mm) FusedSilica Capillaries. Rapid Commun. Mass Spectrom. 1998, 12, 443448.

46. Senko, M. W.; Hendrickson, C. L.; Emmett, M. R.; Shi, S. D.-H.; Marshall, A. G. External Accumulation of Ions for Enhanced Electrospray Ionization Fourier Transform Ion Cyclotron Resonance Mass Spectrometry. J. Am. Soc. Mass Spectrom. 1997, 8, 970-976.

47. Uhlmann, E; Peyman, A; Breipohl, G; Will, D. W. PNA: Synthetic Polyamide Nucleic Acids with Unusual Binding Properties. Angew. Chem. Int. Ed. 1998, 37, 2796-2823.

48. Blake, R. D. (Meyers R.A., ed.) Encylcopedia of Molecular Biology and Molecular Medicine. VCH: New York, 1996. pp 1-19.

49. Hillen, W.; Goodman, T. C.; Wells, R.D. Salt Dependence and Thermodynamic Interpretation of the Thermal Denaturation of Small DNA Restriction Fragments. Nucleic Acids Res. 1981, 9, 415-436.

50. Blake, R. D.; Delcourt, S. G. Thermodynamic Effects of Formamide on DNA Stability. Nucleic Acids Res. 1996, 24, 2095-2103.

51. Falk, M.; Hartman, K. A.; Lord, R. C. Hydration of Deoxyribonucleic Acid II: An Infrared Study. J. Am. Chem. Soc. 1963, 85, 387-391. 